\title{
Three-dimensional analysis of the effects of the treatment on anterior crossbite in the primary dentition
}

\author{
Yasutaka Kaihara, Hideaki Amano, Kazuo Miura* and Katsuyuki Kozai* \\ Department of Pediatric Dentistry, Hiroshima University Hospital \\ * Department of Pediatric Dentistry, Hiroshima University Graduate School \\ 1-2-3 Kasumi, Minami-ku, Hiroshima 734-8553, JAPAN
}

\begin{abstract}
We performed measurement using a 3-dimensional measurement system in study models of children with anterior crossbite in the primary dentition treated using a chin cap and lingual arch, and evaluated changes in the dentition and occlusion after treatment based on measurement values and also using wire frame models. Comparing with the situation before treatment, the overbite of anterior teeth shows improvement to become shallow and the terminal plane becomes vertical type and the occlusal plane becomes flatter. Labioclination of upper anterior teeth, linguoclination of lower anterior teeth and backward movement of lower dentition are observed. Occlusal view shows that both upper and lower dental arch become semicircular configuration. The width, depth and height of the post-treatment dentition became closer to the standard values. Chin cap and lingual arch treatment for anterior crossbite during early childhood is effective, and also helpful in the normal development of occlusion in children.
\end{abstract}

Key words

Anterior crossbite,

Chin cap,

Deciduous dentition,

Occlusion,

Three-dimensional analysis

\section{Introduction}

In pedodontic practice, children with anterior crossbite are frequently encountered, particularly during early childhood ${ }^{1-3)}$. In the treatment of anterior crossbite, the chin cap is sometimes used for the growth inhibition and repositioning of the mandible ${ }^{3-5}$. However, there have been only a few studies that 3-dimensionally analyzed the effects of chin cap and lingual arch treatment on the alignment of the dentition and occlusion. We performed measurement using a 3-dimensional measurement system $^{6,7)}$ in study models of children with anterior crossbite in the primary dentition that diagnosed with mandibular excess with cephalometric analysis treated using a chin cap and lingual arch, and evaluated changes in the dentition and occlusion after treatment based on measurement values and also using wire frame models.

Received on May 26, 2004

Accepted on November 4, 2004

\section{Materials and methods}

\section{Materials}

The materials were study models obtained from 10 Japanese children ( 5 males and 5 females) before and after chin cap treatment for anterior crossbite during the primary dentition period ( 3 to 6 years old). The force magnitude used for therapy ranged from 200 to $500 \mathrm{gm}$ per side of the chin. The mean duration of treatment was 14 months. During the period of chin cap and lingual arch treatment, no patient underwent restoration that may affect occlusion. The chin cap was used in combination with a lingual arch in 7 of the 10 patients.

\section{Determination and expression methods of measurement points}

The following measurement points were established: incisal edge of the deciduous incisor, the cusp tops of the deciduous canine and molar, mesial and distal occlusocervical convexities of the tooth crown, the lowest point of the labial cervical line in each tooth, 


\section{Deciduous Incisor}

Deciduous canine

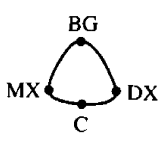

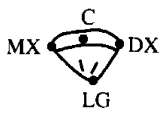

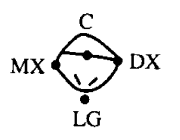

\section{Deciduous Molor}

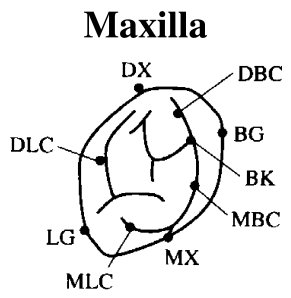

Mandible

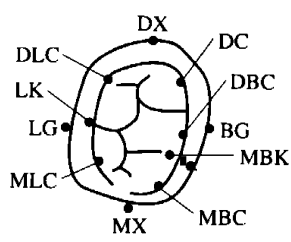

M: mesial D: distal B: buccal L: lingual $\mathrm{C}$ : the center of incisal edge or cusp tip MX: mesial occlusocervical convexity of tooth crown DX: distal occlusocervical convexity of tooth crown BG: the lowest point of labial cervical line LG: the lowest point of lingual cervical line

Fig. 1 Measuring points

and the lowest point of the lingual cervical line in each tooth. Anatomical sites were expressed in terms of alphabets (Fig. 1). The measurement points were expressed in terms of the tooth number followed by alphabets.

Example 1) $65 \mathrm{MBC} \cdots$ mesio-buccal cusp top of the maxillary deciduous left second molar

Example 2) $81 \mathrm{C} \cdots$ center of the incisal edge of the mandibular deciduous right central incisor

\section{Measurement and data processing methods}

A 3-dimensional model measurement system was used. This system consists of a measuring device by the direct method and a personal computer that controls the device ${ }^{6}$. The accuracy of the measuring device is about $0.1 \mathrm{~mm}$ for $\mathrm{X}$ and $\mathrm{Z}$ axes and $0.05 \mathrm{~mm}$ for $\mathrm{Y}$ axis. The accuracy of model measuring is less than $0.1 \mathrm{~mm}$. In addition, this system allows the reproduction of the occlusal relationship between the upper and lower dental arches by computer simulation. The error for the reproduction of the occlusal relationship is within $0.1 \mathrm{~mm}$. Model measurement and the reproduction of the occlusal relationship were performed by the following procedures.

1) All measurement points determined on the model were input separately for the maxilla and mandible.

2) The maxillomandibular model was fixed in the articulated position, and the lowest point of the labial cervical line (hereafter, BG) in each tooth in the maxilla and mandible was re-inputted.

3 ) The coordinate conversion matrix was optimized from BG data in 1) and 2), and all data in 1) were converted to the coordinate of 2 ) by the following method.

We assumed that the model is a solid body, and its position moves after articulation. The BG data matrix in the maxilla and that in the mandible before articulation were defined as $\boldsymbol{p}_{u}$ and $\boldsymbol{p}_{l}$, respectively, and the BG data matrix in the maxilla and that in the mandible after articulation were defined as $\boldsymbol{p}_{u}{ }_{u}$ and $\boldsymbol{p}_{l}{ }_{l}$, respectively. The relationship between $\boldsymbol{p}_{u}$ and $\boldsymbol{p}_{l}$ and that between $\boldsymbol{p}_{u}^{\prime}$ and $\boldsymbol{p}_{l}^{\prime}$, after adding measurement errors $\boldsymbol{N}_{u}$ and $\boldsymbol{N}_{l}$, could be expressed using the following formula.

$$
\begin{aligned}
& \boldsymbol{p}_{u}^{\prime}=\boldsymbol{R}_{u} \boldsymbol{p}_{u}+\boldsymbol{T}_{u}+\boldsymbol{N}_{u}, \boldsymbol{p}_{l}^{\prime}=\boldsymbol{R}_{l} \boldsymbol{p}_{l}+\boldsymbol{T}_{l}+\boldsymbol{N}_{l} \\
& \left(\boldsymbol{R}_{u}, \boldsymbol{R}_{l} \text { : rotation matrix; } \boldsymbol{T}_{u}, \boldsymbol{T}_{l} \text { : translation vector }\right)
\end{aligned}
$$

Estimating the articulated position based on the results of 1) and 2) ends in optimizing the error model produced by the rotation matrix, translation vector, and noise vector. Therefore, to obtain the estimated values of the rotation matrix and the translation vector, we defined a functional by the square sum of Euclideam norm and adopted the least square method using single value resolution for the optimization of the error model ${ }^{8)}$. The algorithm was as follows.

$<$ Problem $>$

Two 3-D point sets $\left\{\boldsymbol{p}_{i}\right\},\left\{\boldsymbol{p}_{i}^{\prime}\right\}\left(\left\{\boldsymbol{p}_{i}\right\},\left\{\boldsymbol{p}_{i}^{\prime}\right\}: 3 \times 1\right.$ column matrices)

$$
\Sigma^{2}=\sum_{i=1}^{N}\left|\boldsymbol{p}^{\prime}-(\boldsymbol{R} \boldsymbol{p}+\boldsymbol{T}+\boldsymbol{N})\right|^{2}
$$

$(\boldsymbol{R}$ : Rotation matrix; $\boldsymbol{T}$ : Translation vector;

$N$ : Noise vector)<smiles>[AlH2]</smiles>

$\underset{\downarrow}{\operatorname{minimize}} \Sigma^{2}$

finding $\hat{\boldsymbol{R}}$ and $\hat{\boldsymbol{T}}$

$<$ Algorithm for finding Rotation and Translation $>$ A. Finding $\hat{\boldsymbol{R}}$

i) calculate $\boldsymbol{q}=\frac{1}{N} \sum_{i=1}^{N} \boldsymbol{p}_{i}$ and $\boldsymbol{q}^{\prime}=\frac{1}{N} \sum_{i=1}^{N} \boldsymbol{p}_{i}$

ii) calculate $\boldsymbol{r}_{i}=\boldsymbol{p}_{i}-\boldsymbol{q}$ and $\boldsymbol{r}_{i}^{\prime}=\boldsymbol{p}_{i}^{\prime}-\boldsymbol{q}^{\prime}$ 


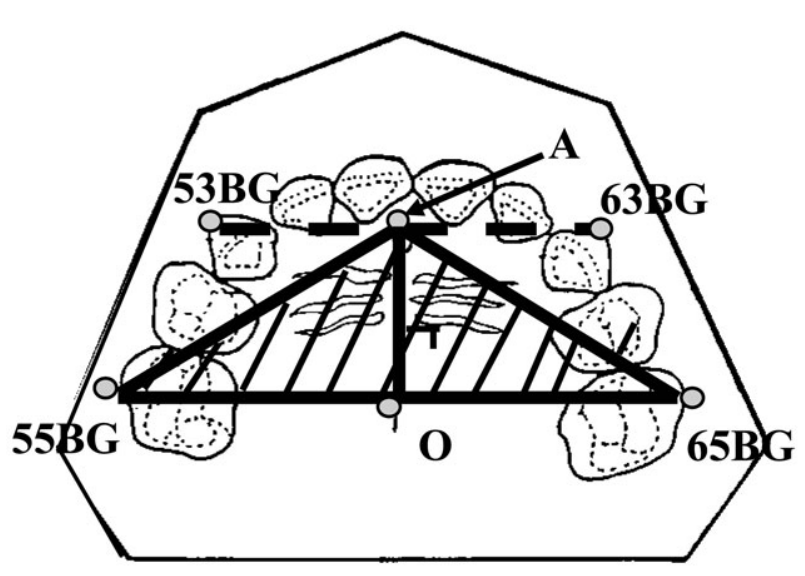

A: The mid point of the straight line formed by connecting $53 \mathrm{BG}$ and $63 \mathrm{BG}$

$\mathrm{O}$ : Origin

AIIm: Reference plane

Fig. 2 Reference plane

iii) calculate $\boldsymbol{H}=\sum_{i=1}^{N} \boldsymbol{r}_{i} \boldsymbol{r}_{i}$

iv) Find the singular value decomposition of $\boldsymbol{H}$ $\boldsymbol{H}=\boldsymbol{U} \Lambda \boldsymbol{V}^{t}$

v) calculate $\boldsymbol{R}=\boldsymbol{V} \boldsymbol{U}^{t}$

B. Finding $\hat{\boldsymbol{T}}$ calculate $\hat{\boldsymbol{T}}=\boldsymbol{q}^{\prime}-\hat{\boldsymbol{R}} \boldsymbol{q}$

4) Data obtained by data processing in 3) were converted to the coordinate system determined by the reference plane that contains $55 \mathrm{BG}, 65 \mathrm{BG}$, and the midpoint of the line connecting 53BG and $63 \mathrm{BG}$ and uses the foot of the perpendicular from this midpoint to the line connecting 55BG and $65 \mathrm{BG}$ as the origin (Fig. 2). Thus, the final data matrix was obtained.

5) After numerical processing and image processing of the final data matrix, the arch and occlusion were analyzed.

\section{Analysis based on coordinate values and the distances between measurement points}

\section{a) Arch width}

The following distances were calculated as arch width: distances between the cusps of the bilateral deciduous canines in the maxilla and mandible, distances between the lowest lingual cervical points of the bilateral deciduous canines, deciduous first molars, and deciduous second molars in the maxilla

and mandible, the distances between the lingual cusps of the bilateral deciduous first molars and deciduous second molars in the maxilla and mandible, distance between the buccal terminal sulci of the mandibular deciduous first molars, and the distance between the mesiobuccal terminal sulci of the deciduous second molars.

b) Arch length

As arch length, the lengths of perpendiculars from the midpoint of the mesial occlusocervical convexities of the bilateral deciduous central incisors to the following lines were calculated: the line connecting the bilateral cusps of the deciduous canines (1-3C), line connecting the buccal terminal sulci of the bilateral deciduous second molars (1-5B), and the line connecting the distal occlusocervical convexities of the bilateral deciduous second molars (1-5D).

c) Overbite and overjet

The vertical and horizontal distances from $61 \mathrm{C}$ and $71 \mathrm{C}$ to the reference plane were calculated as overbite and overjet, respectively.

d) Occlusal curvature

Occlusal curvature was defined as the curve obtained by linking Y values of $\mathrm{C}, \mathrm{MBC}$, and $\mathrm{DBC}$ from the reference plane.

e) Dental height

Dental height was defined as the absolute value of the difference of the $\mathrm{Y}$ value of the lowest point of the labial cervical line in each tooth.

\section{Analysis based on profilograms}

Arches and occlusion were analyzed based on profilograms obtained by converting measurement data to images using wire frame models. Profilograms were produced by connecting measurement points as follows.

a) Frontal view and lateral view

In the anterior teeth, the center of the incisal edge (C), mesial occlusocervical convexity of the tooth crown (MX), the lowest point of the labial cervical line $(\mathrm{BG})$, and the distal occlusocervical convexity of the tooth crown (DX) were connected. In the canines, the cusp top (C), mesial occlusocervical convexity of the tooth crown (MX), the lowest point of the labial cervical line (BG), and the distal occlusocervical convexity of the tooth crown (DX) were connected. In the deciduous molars, mesio-buccal cusp (MBC), mesial occlusocervical convexity of the tooth crown (MX), the lowest point of the labial cervical line (BG), and the distal 
Table 1 The values of arch width

\begin{tabular}{|c|c|c|c|c|c|c|c|c|c|c|c|c|c|c|c|c|c|c|c|}
\hline & & \multicolumn{6}{|c|}{ Deciduous canine } & \multicolumn{6}{|c|}{ First deciduous molar } & \multicolumn{6}{|c|}{ Second deciduous molar } \\
\hline & & \multicolumn{2}{|c|}{$\mathrm{C}$} & \multicolumn{2}{|c|}{ LG } & \multicolumn{2}{|c|}{$\mathrm{BG}$} & \multicolumn{2}{|c|}{$\mathrm{LC}$} & \multicolumn{2}{|c|}{ B } & \multicolumn{2}{|c|}{ LG } & \multicolumn{2}{|c|}{$\mathrm{LC}$} & \multicolumn{2}{|c|}{ B } & \multicolumn{2}{|c|}{ LG } \\
\hline & & before & after & before & after & before & after & before & after & before & after & before & after & before & after & before & after & before & after \\
\hline \multirow{3}{*}{ Maxilla } & $\mathrm{N}$ & 10 & 10 & 10 & 10 & 10 & 10 & 10 & 10 & & & 10 & 10 & 10 & 10 & 10 & 10 & 10 & 10 \\
\hline & MEAN & 29.97 & 30.52 & 24.79 & 25.35 & 39.25 & 40.15 & 31.30 & 32.48 & & & 27.16 & 28.46 & 35.15 & 36.64 & 45.53 & 47.39 & 29.44 & 31.35 \\
\hline & S.D. & 1.16 & 1.57 & 1.52 & 2.02 & 1.69 & 2.20 & 2.05 & 2.35 & & & 1.75 & 2.31 & 2.27 & 2.53 & 2.76 & 2.78 & 2.38 & 3.00 \\
\hline \multirow[t]{2}{*}{ Mandible } & MEAN & 23.10 & 22.82 & 19.53 & 19.54 & 30.46 & 30.66 & 27.19 & 26.99 & 33.23 & 33.43 & 24.43 & 24.57 & 31.08 & 31.23 & 39.79 & 39.79 & 28.71 & 29.32 \\
\hline & S.D. & 1.11 & 1.03 & 1.05 & 1.02 & 1.50 & 1.12 & 1.15 & 1.08 & 1.17 & 1.10 & 0.77 & 0.96 & 1.29 & 1.25 & 1.08 & 1.63 & 1.22 & 1.16 \\
\hline
\end{tabular}

C: The width of cusp of deciduous canine in both side LC: The width of cusp of deciduous molars in both side $\mathrm{B}$ : The width of left and right buccal terminal sulci of second deciduous molars

BG: The width of cusp of buccal cervical line in both side LG: The width of lingual cervical line in both side before: before treatment after: after treatment

Table 2 The values of arch length

\begin{tabular}{ccccccccc}
\hline & \multicolumn{2}{c}{$1-3 \mathrm{C}$} & \multicolumn{2}{c}{$1-5 \mathrm{~B}$} & \multicolumn{2}{c}{$1-5 \mathrm{D}$} \\
& & before & after & before & after & before & after \\
\hline \multirow{3}{*}{ Maxilla } & MEAN & 5.65 & 6.88 & 19.79 & 20.75 & 24.96 & 26.18 \\
& S.D. & 0.82 & 0.90 & 0.82 & 1.08 & 0.71 & 1.12 \\
\hline \multirow{2}{*}{ Mandible } & N & 10 & 10 & 10 & 10 & 10 & 10 \\
& MEAN & 4.64 & 4.18 & 18.60 & 17.52 & 25.20 & 24.03 \\
& S.D. & 0.96 & 1.13 & 1.02 & 1.26 & 1.16 & 1.17 \\
\hline & & & & & $*: P<0.05$ & $* *: P<0.01 \quad(\mathrm{~mm})$
\end{tabular}

1-3C: The length of straight line formed by connecting the mid point of mesial occlusocervical convexity of central deciduous incisor with cusp of deciduous canine in both side

1-5B: The length of straight line connecting left and right buccal terminal sulci of second deciduous molars

1-5D: The length of perpendicular to the straight line formed by connecting left and right distal occlusocervical convexity of second deciduous molar crown

before: before treatment after: after treatment

Table 3 Overbite and overjet

\begin{tabular}{ccccc}
\hline & \multicolumn{2}{c}{ overbite } & \multicolumn{2}{c}{ overjet } \\
& before & after & before & after \\
\hline $\mathrm{N}$ & 10 & 10 & 10 & 10 \\
MEAN & 1.74 & 0.06 & -2.11 & 1.44 \\
S.D. & 1.15 & 0.66 & 0.53 & 1.10 \\
\hline & & & $* * *: P<0.001 \quad(\mathrm{~mm})$
\end{tabular}

before: before treatment after: after treatment

occlusocervical convexity of the tooth crown (DX) were connected. Therefore, each tooth was expressed as a trapezium. In addition, the lowest points of the labial cervical lines (BG) of all teeth were connected, and the incisal edges $(\mathrm{C})$, cusps of the canines (C) and the mesio-buccal cusps (MBC) were similarly connected.

b) Occlusal view

Each tooth was expressed as a trapezium formed by connecting MX, BG, DX, and LG of each tooth. To make the alignment of each tooth clearly, MX and DX in each tooth were connected, and BG and LG were connected.

c) Superimposition of after treatment profilograms on before treatment ones

After treatment profilograms were superimposed on before treatment ones and analyzed. 


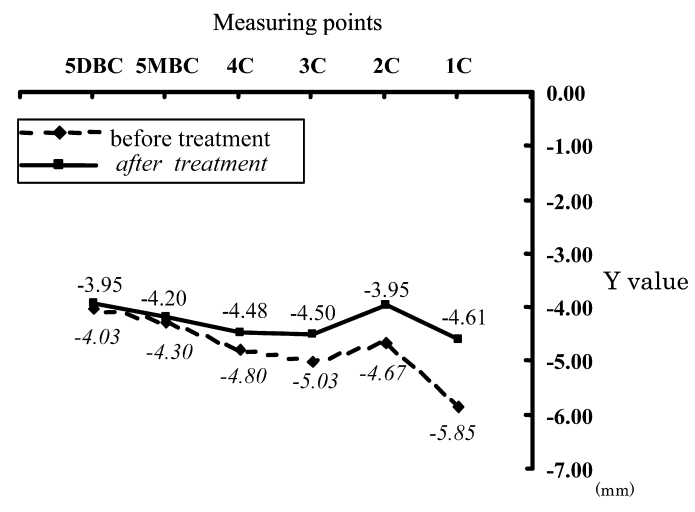

Fig. 3 Occlusal curvature (Maxilla)

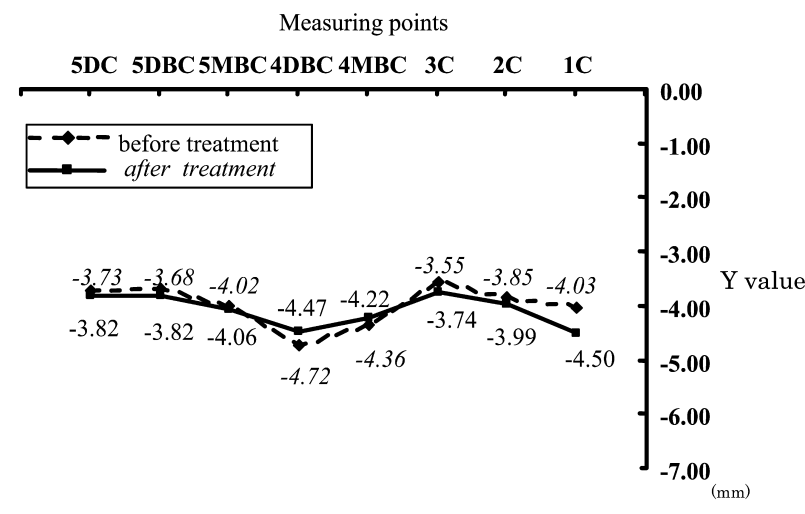

Fig. 4 Occlusal curvature (Mandible)

1: deciduous central incisor 2: deciduous lateral incisor 3: deciduous canine 4: deciduous first molar 5: deciduous second molar M: mesial D: distal B: buccal L: lingual C: the center of incisal edge or cusp tip

Table 4 The values of dental height:

Dental height is the absolute value of difference of $\mathrm{Y}$ values at the lowest point of labial cervical line in each tooth.

\begin{tabular}{cccccccccccc}
\hline & \multicolumn{2}{c}{$\begin{array}{c}\text { Central incisor } \\
\text { before }\end{array}$} & \multicolumn{2}{c}{ Lafteral incisor } & \multicolumn{2}{c}{ Deciduous canine } & \multicolumn{2}{c}{ First } & \multicolumn{2}{c}{$\begin{array}{c}\text { Second } \\
\text { deciduous molar }\end{array}$} & \multicolumn{2}{c}{ deciduous molar } \\
before & after & before & after & before & after & before & after \\
\hline N & 10 & 10 & 10 & 10 & 10 & 10 & 10 & 10 & 10 & 10 \\
MEAN & 7.46 & 8.21 & 8.22 & 8.58 & 9.04 & 8.88 & 8.78 & 8.95 & 7.67 & 7.75 \\
S.D. & 1.50 & 1.11 & 1.17 & 1.06 & 1.46 & 1.39 & 1.22 & 1.39 & 0.83 & 1.01 \\
\hline
\end{tabular}

before: before treatment after: after treatment

$(\mathrm{mm})$

\section{Results}

\section{Analysis based on coordinate values and distances between measurement points}

Table 1 shows the values of arch width. In the maxilla, the values of arch width were higher in all measurement points after treatment than before treatment. In the mandible, the values of arch width were similar between before and after treatment.

Table 2 shows the values of arch length. In the maxilla, the values of arch length were significantly higher in all measurement points after treatment than before treatment $(P<0.05)$. In the mandible, the values of arch length were shorter after treatment than before treatment showing significant differences for $1-5 B$ and $1-5 D$, but not for $1-3 C$. Thus, after treatment, the maxillary arch length increased while the mandibular arch length decreased.

After treatment, overbite significantly decreased, and overjet significantly increased (Table 3 ).

Figure 3 and Fig. 4 show the occlusal curvatures of the maxilla and mandible, respectively. Since the occlusal curvature did not significantly difference between the right and left sides in the maxilla or mandible, the mean of the values on both sides was calculated. In the maxilla, the maximum and minimum values before treatment were -4.03 and $-5.85 \mathrm{~mm}$, respectively, with the difference being $1.82 \mathrm{~mm}$, but those after treatment were -3.95 and $-4.61 \mathrm{~mm}$ with the difference being $0.66 \mathrm{~mm}$. In the mandible, the maximum and minimum values before treatment were -3.55 and $-4.72 \mathrm{~mm}$, respectively, with the difference being $1.17 \mathrm{~mm}$, but those after treatment were -3.74 and $-4.47 \mathrm{~mm}$ with the difference being $0.73 \mathrm{~mm}$. Therefore, the difference between the maximum and minimum values decreased after treatment in both the maxilla and mandible, indicating flattening of the occlusal curvature after treatment.

The values of dental height increased after treatment in the deciduous anterior teeth, but did not change in the deciduous canines or molars (Table 4). 

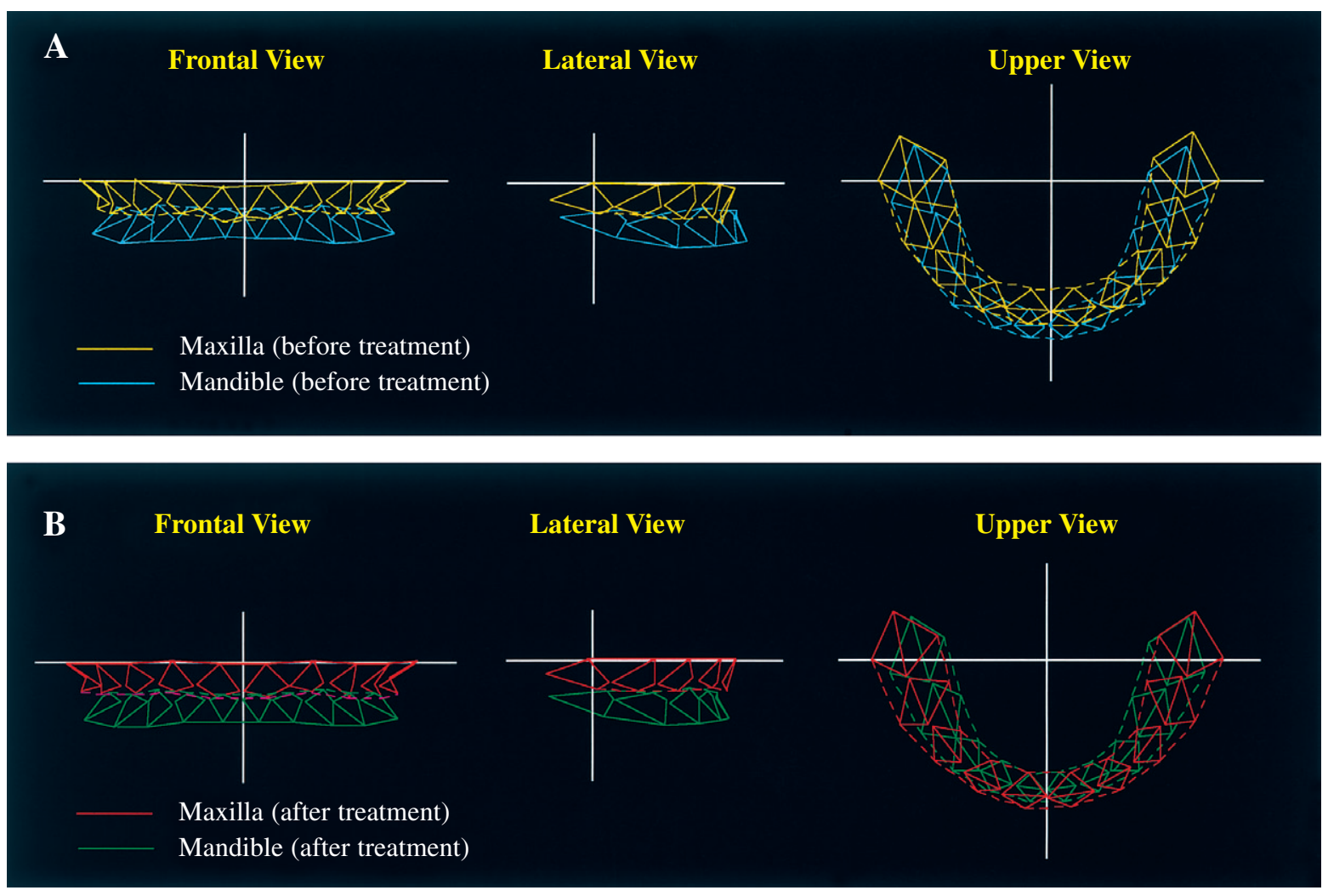

Fig. 5 Profilograms of before treatment and after treatment
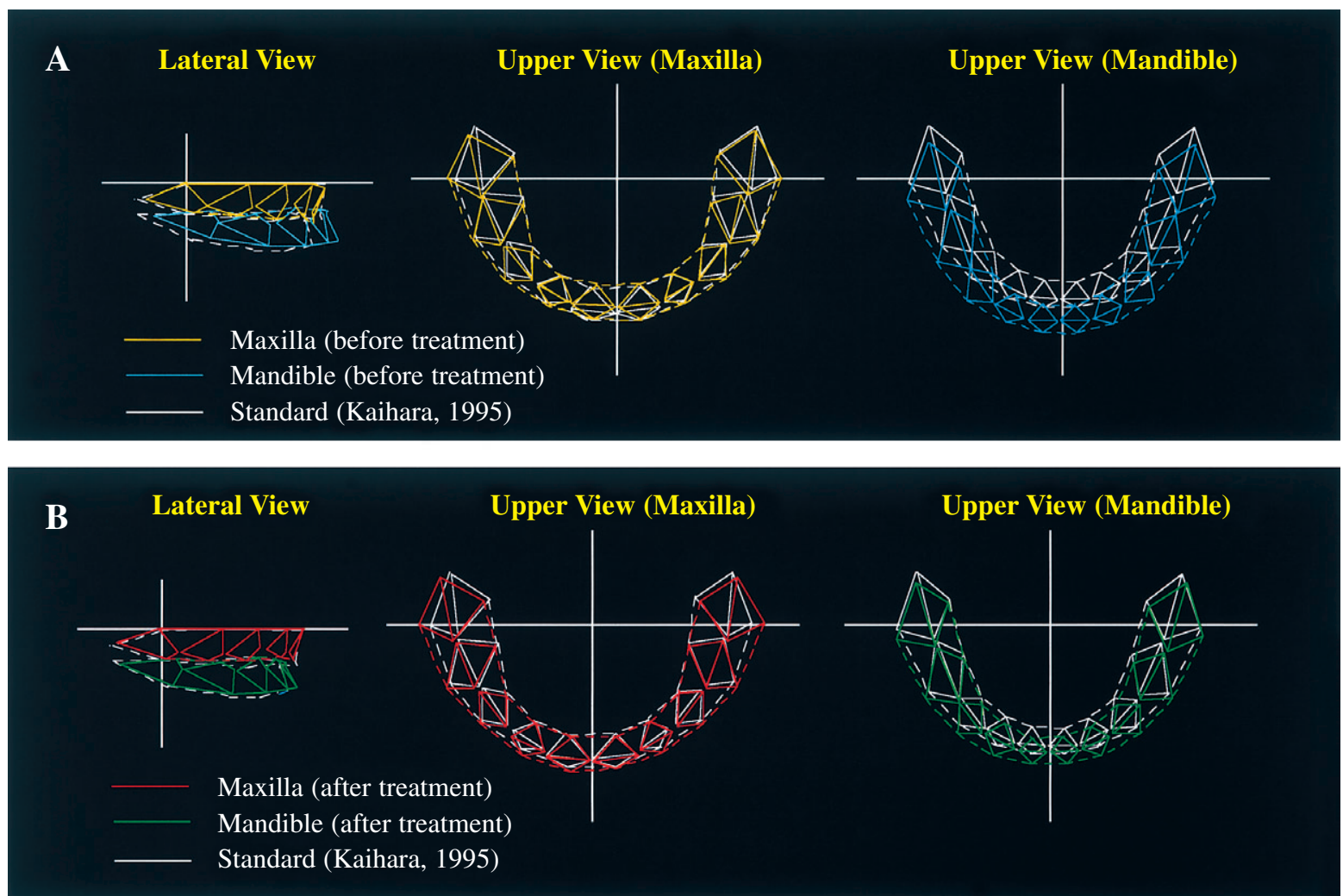

Fig. 6 Superimposition of Profilograms 


\section{Analysis based on profilograms}

Profilograms before treatment are shown in Fig. 5A. A frontal view revealed deep overbite but no median discrepancy. A lateral view showed lingual inclination of the maxillary anterior teeth, labial inclination of the mandibular anterior teeth, a large curve of Spee, and a mesial step type terminal plane. An occlusal view showed a mesial step type terminal plane, mandibular anterior teeth protruding over the maxillary anterior teeth, but no bilateral asymmetry of the arch or median discrepancy.

Figure 5B shows profilograms after treatment. After treatment, overbite improved, becoming shallower, the terminal plane became the vertical type, and the curve of Spee was flattened. In the maxilla, labial inclination of the anterior teeth was observed. In the mandible, posterior transfer of the arch and lingual inclination of the anterior teeth were observed. An occlusal view revealed that the arch became semicircular after treatment in both maxilla and mandible. Figure 6 shows before treatment profilograms superimposed on standard value profilograms and after treatment profilograms superimposed on standard value profilograms, respectively. After treatment, the arch approached to the standard arch in terms of width, depth, and height.

\section{Discussion}

Concerning the occlusal relationship between the maxillary and mandibular dental arches, the terminal plane changed from the mesial step type to the vertical type, and anteroposterior overlapping and deep bite in the anterior teeth improved after treatment. After treatment, in the maxilla, an increase in arch length, flattening of the occlusal curvature, and values of arch shape closer to the standard values. In the mandibular, lack of changes in arch width, posterior transfer of the arch, a decrease in arch length, flattening of the occlusal curvature, and values of arch shape closer to the standard values. These results suggest that the arch shape and maxillomandibular occlusal relationship approached normal.

Ishikawa et al. ${ }^{9)}$ reported that the lingual inclination of mandibular anterior teeth and crowding in mandibular permanent anterior teeth between lower left and right deciduous canine are often observed when a chin cap was used for anterior crossbite in the deciduous dentition or early mixed dentition stage. In this study, we evaluated the dentition and occlusion until eruption of permanent teeth, but none developed crowding in the permanent anterior teeth. However, the arch width between lower left and right deciduous canine did not increase after treatment, and lingual inclination of the mandibular anterior teeth was observed. Thus, attention should be paid to the possible development of crowding in the mandibular permanent anterior teeth when a chin cap was used to treat anterior crossbite in the deciduous dentition.

The usefulness of the chin cap for treating anterior crossbite has been established. Mandibular growth suppression and posterior transfer after chin cap treatment have been reported ${ }^{10)}$. More marked effects of chin cap treatment at lower age have been also reported ${ }^{11)}$. Tsuchikawa et al. ${ }^{10)}$ described that no changes in skeletal profile occur when chin cap treatment was initiated at the age of about 10 years.

Nukatsuka ${ }^{11)}$ reported changes in shape, size and position of the mandible after chin cap treatment and more marked effects at lower age. Oda et al. ${ }^{12)}$ also recommended chin cap treatment at lower age. Another study suggested that the chin cap does not suppress mandibular growth but only affects the growth process during its use ${ }^{13)}$.

However, chin cap treatment may eliminate factors suppressing mandibular anterior growth, even if it does not suppress mandibular growth, and may decrease the possibility that an Angle class III relationship develops between the upper and lower first permanent molar, or the mandible grows anteroposteriorly while remaining in the forwarded position.

In conclusion, our study found that chin cap and lingual arch treatment for anterior crossbite during early childhood is effective, and also helpful in the normal development of occlusion in children.

\section{References}

1) Nagata, M., Tanaka, K., Hayasaki, H., Watanabe, R., Yamasaki, Y. and Nakata, M.: An investigation on the patients records of the first visit in Pediatric Dental Clinic of Kyusyu Dental Hospital. Jpn J Ped Dent 33: 543-551, 1995. (in Japanese, English abstract)

2) Shiono, K., Shimizu, H. and Ogura, T.: An investigation on occlusal guidance patients at the Pediatric Department of Kagoshima University. Jpn J Ped Dent 31: 62-68, 1993. (in Japanese, English abstract) 
3) Yamasaki, K., Hirota, K., Yamasaki, Y., Nonaka, K. and Nakata, M.: An investigation into the actual condition of patients with occlusal disharmony at the Pediatric Dental Clinic of Kyusyu Dental Hospital. Jpn J Ped Dent 27: 522-528, 1989. (in Japanese, English abstract)

4) Suzuki, N.: A cephalometric observation on the effect of chin cap. Orthod Waves 31: 64-74, 1972. (in Japanese, English abstract)

5) Irie, M. and Nakamura, S.: Orthopedic approach to severe skeletal Class III malocclusion. Am J Orthod 67: 377-392, 1975.

6) Kaihara, Y.: A study on the development process of dentition and occlusion in the Japanese children with the three dimensional measurement system of dental casts. J Hiroshima Univ Dent Soc 27: 69-98, 1995. (in Japanese)

7) Kaihara, Y., Amano, H., Miura, K. and Nagasaka, N.: A study of a new method for diagnosis of the dentition and occlusion in children with a threedimensional measurement system of dental casts. Jpn J Ped Dent 36: 839-847, 1998. (in Japanese, English abstract)

8) Arun, K.S., Huang, T.S. and Blostein, S.D.: Least- squares fitting of two 3-D point sets. IEEE Trans PAMI 9: 698-700, 1987.

9) Ishikawa, M., Xiaoling, S., Saitoh, M. and Funayama, K.: A clinical study of lower permanent anterior crowding and its easement after treatment of anterior cross bite in the deciduous dentition. Jpn J Ped Dent 33: 525-535, 1985. (in Japanese, English abstract)

10) Tsuchikawa, T., Sugawara, J., Nakamura, H. and Mitani, H.: Long-term results of skeletal profile changes occurred in the chin cap therapy of Japanese male skeletal III cases. Orthod Waves 44: 644-659, 1985. (in Japanese, English abstract)

11) Nukatsuka, S.: The longitudinal study of orthopedic effect caused by chin cap treatment. Tohoku University Dental Journal 1: 1-17, 1982. (in Japanese)

12) Oda, H., Ueda, A., Miyagawa, Y., Hongou, H., Kuroki, K., Suzuki, M. and Suzuki, Y.: An individual growth study of the effects of chin cap force to the mandible. Orthod Waves 48: 355-361, 1989. (in Japanese, English abstract)

13) Morinushi, T., Kishimoto, T., Miyagawa, T. and Ogura, T.: Re-examination in regard to the application of chin cap. Jpn J Ped Dent 36: 604-612, 1998. (in Japanese, English abstract) 